\title{
Discovery of VHE $\gamma$-ray emission from the BL Lacertae object B3 2247+381 with the MAGIC telescopes
}

J. Aleksić ${ }^{1}$, E. A. Alvarez ${ }^{2}$, L. A. Antonelli ${ }^{3}$, P. Antoranz ${ }^{4}$, M. Asensio ${ }^{2}$, M. Backes $^{5}$, J. A. Barrio ${ }^{2}$, D. Bastieri ${ }^{6}$, J. Becerra González ${ }^{7,8}$, W. Bednarek ${ }^{9}$, A. Berdyugin ${ }^{10}$, K. Berger ${ }^{7,8}$, E. Bernardini ${ }^{11}$, A. Biland ${ }^{12}$, O. Blanch ${ }^{1}$, R. K. Bock ${ }^{13}$, A. Boller ${ }^{12}$, G. Bonnoli ${ }^{3}$, D. Borla Tridon ${ }^{13}$, I. Braun ${ }^{12}$, T. Bretz ${ }^{14,26}$, A. Cañellas ${ }^{15}$, E. Carmona ${ }^{13}$, A. Carosi ${ }^{3}$, P. Colin ${ }^{13}$, E. Colombo ${ }^{7}$, J. L. Contreras ${ }^{2}$, J. Cortina ${ }^{1}$, L. Cossio ${ }^{16}$, S. Covino ${ }^{3}$, F. Dazzi ${ }^{16,27}$, A. De Angelis ${ }^{16}$, G. De Caneva ${ }^{11}$, E. De Cea del Pozo ${ }^{17}$, B. De Lotto ${ }^{16}$, C. Delgado Mendez ${ }^{7,28}$, A. Diago Ortega ${ }^{7,8}$, M. Doert ${ }^{5}$, A. Domínguez ${ }^{18}$, D. Dominis Prester ${ }^{19}$, D. Dorner ${ }^{12}$, M. Doro ${ }^{20}$, D. Elsaesser ${ }^{14}$, D. Ferenc ${ }^{19}$, M. V. Fonseca ${ }^{2}$, L. Font ${ }^{20}$, C. Fruck ${ }^{13}$, R. J. García López ${ }^{7,8}$, M. Garczarczyk ${ }^{7}$, D. Garrido ${ }^{20}$, G. Giavitto ${ }^{1}$, N. Godinović ${ }^{19}$, D. Hadasch ${ }^{17}$, D. Häfner ${ }^{13}$, A. Herrero ${ }^{7,8}$, D. Hildebrand ${ }^{12}$, D. Höhne-Mönch ${ }^{14}$, J. Hose ${ }^{13}$,

D. Hrupec ${ }^{19}$, B. Huber ${ }^{12}$, T. Jogler ${ }^{13}$, H. Kellermann ${ }^{13}$, S. Klepser ${ }^{1}$, T. Krähenbühl ${ }^{12}$, J. Krause ${ }^{13}$, A. La Barbera ${ }^{3}$, D. Lelas ${ }^{19}$, E. Leonardo ${ }^{4}$, E. Lindfors ${ }^{10}$, S. Lombardi ${ }^{6}$, M. López ${ }^{2}$, A. López-Oramas ${ }^{1}$, E. Lorenz ${ }^{12,13}$, M. Makariev ${ }^{21}$, G. Maneva ${ }^{21}$, N. Mankuzhiyil ${ }^{16}$, K. Mannheim ${ }^{14}$, L. Maraschi ${ }^{3}$, M. Mariotti ${ }^{6}$, M. Martínez ${ }^{1}$, D. Mazin ${ }^{1,13}$, M. Meucci ${ }^{4}$, J. M. Miranda ${ }^{4}$, R. Mirzoyan ${ }^{13}$, H. Miyamoto ${ }^{13}$, J. Moldón ${ }^{15}$, A. Moralejo ${ }^{1}$, P. Munar-Adrover ${ }^{15}$, D. Nieto ${ }^{2}$, K. Nilsson ${ }^{10,29}$, R. Orito ${ }^{13}$, I. Oya ${ }^{2}$, D. Paneque ${ }^{13}$, R. Paoletti ${ }^{4}$, S. Pardo ${ }^{2}$, J. M. Paredes ${ }^{15}$, S. Partini ${ }^{4}$, M. Pasanen ${ }^{10}$, F. Pauss ${ }^{12}$, M. A. Perez-Torres ${ }^{1}$, M. Persic ${ }^{16,22}$, L. Peruzzo ${ }^{6}$, M. Pilia ${ }^{23}$, J. Pochon ${ }^{7}$, F. Prada ${ }^{18}$, P. G. Prada Moroni ${ }^{24}$, E. Prandini ${ }^{6}$, I. Puljak ${ }^{19}$, I. Reichardt ${ }^{1}$, R. Reinthal ${ }^{10}$, W. Rhode ${ }^{5}$, M. Ribó ${ }^{15}$, J. Rico ${ }^{25,1^{\prime}}$, S. Rügamer ${ }^{14}$, A. Saggion ${ }^{6}$, K. Saito ${ }^{13}$, T. Y. Saito ${ }^{13}$, M. Salvati ${ }^{3}$, K. Satalecka ${ }^{2}$, V. Scalzotto ${ }^{6}$, V. Scapin ${ }^{2}$, C. Schultz ${ }^{6}$, T. Schweizer ${ }^{13}$, M. Shayduk ${ }^{13}$, S. N. Shore ${ }^{24}$, A. Sillanpää ${ }^{10}$, J. Sitarek ${ }^{9}$, I. Snidaric ${ }^{19}$, D. Sobczynska ${ }^{9}$, F. Spanier ${ }^{14}$, S. Spiro ${ }^{3}$, V. Stamatescu ${ }^{1}$, A. Stamerra ${ }^{4}$, B. Steinke ${ }^{13}$, J. Storz ${ }^{14}$, N. Strah ${ }^{5}$, T. Surić ${ }^{19}$, L. Takalo ${ }^{10}$, H. Takami ${ }^{13}$, F. Tavecchio ${ }^{3}$, P. Temnikov ${ }^{21}$, T. Terzić ${ }^{19}$, D. Tescaro ${ }^{24}$, M. Teshima ${ }^{13}$, O. Tibolla ${ }^{14}$, D. F. Torres ${ }^{25,17}$, A. Treves ${ }^{23}$, M. Uellenbeck ${ }^{5}$, H. Vankov ${ }^{21}$, P. Vogler ${ }^{12}$, R. M. Wagner ${ }^{13}$, Q. Weitzel ${ }^{12}$, V. Zabalza ${ }^{15}$, F. Zandanel $^{18}$, R. Zanin $^{1}$ V. Kadenius ${ }^{10}$, M. Weidinger ${ }^{14}$, and S. Buson ${ }^{30,31}$

(Affiliations can be found after the references)

Received 29 August 2011 / Accepted 25 November 2011

\section{ABSTRACT}

Aims. We study the non-thermal jet emission of the BL Lac object B3 2247+381 during a high optical state.

Methods. The MAGIC telescopes observed the source during 13 nights between September 30th and October 30th 2010, collecting a total of $14.2 \mathrm{~h}$ of good quality very high energy (VHE) $\gamma$-ray data. Simultaneous multiwavelength data was obtained with X-ray observations by the Swift satellite and optical $R$-band observations at the KVA-telescope. We also use high energy $\gamma$-ray $(\mathrm{HE}, 0.1-100 \mathrm{GeV})$ data from the Fermi satellite. Results. The BL Lac object B3 2247+381 $(z=0.119)$ was detected, for the first time, at VHE $\gamma$-rays at a statistical significance of 5.6 $\sigma$. A soft VHE spectrum with a photon index of $-3.2 \pm 0.6$ was determined. No significant short term flux variations were found. We model the spectral energy distribution using a one-zone SSC-model, which can successfully describe our data.

Key words. galaxies: active - BL Lacertae objects: individual: B3 2247+381

\section{Introduction}

The number of known extragalactic very high energy (VHE, $E>100 \mathrm{GeV}) \gamma$-ray emitters has increased from 6 to almost 50 (July 2011) during the past five years ${ }^{1}$. Most of these objects are active galactic nuclei (AGN), especially blazars (flat spectrum radio quasars and BL Lac objects), which are known for their large variability across the electromagnetic spectrum from radio to VHE $\gamma$-rays. Eight of these new discoveries were made during high optical states of these sources, reported by the Tuorla blazar monitoring program, which have triggered MAGIC observations: Mrk 180 (Albert et al. 2006),

\footnotetext{
${ }^{1}$ http://www.mpp.de/ rwagner/sources/
}

1ES 1011+496 (Albert et al. 2007a), BL Lacertae (part of the data taken as ToO due to optical high state, Albert et al. 2007b), 3C 279 (Albert et al. 2008a; Aleksić et al. 2011a), MAGIC J0223+430 (Aliu et al. 2009), S5 0716+714 (Anderhub et al. 2009), B3 2247+381 (Mariotti et al. 2010, this paper) and most recently 1ES 1215+303 (Mariotti et al. 2011). This suggests that high optical states are an indication of a higher state also in VHE $\gamma$-ray band, at least in some sources. Additionally for PG $1553+113$ a long term study (Aleksić et al., in prep.) suggests a correlation between the optical and VHE $\gamma$-ray flux, while for PKS 2155-304 it seems that, at least, in some cases the two wavebands are correlated (Foschini et al. 2007; Aharonian et al. 2009). 
The object B3 2247+381 was selected from the sample presented in Nieppola et al. (2006) with a reported X-ray flux $F(>1 \mathrm{keV})>2$ mJy. Donato et al. (2001) classify it as highenergy-peaked BL Lac object and the reported X-ray flux of the source is $F(>1 \mathrm{keV})=0.6 \mathrm{mJy}^{2}$. B3 2247+381 $(z=0.119$, Falco et al. 1988) has been previously observed by the MAGIC telescope as part of the systematic search of VHE $\gamma$-rays from X-ray bright BL Lac objects (Aleksić et al. 2011b) ${ }^{3}$. MAGIC observed the source between August and September 2006, which resulted in an upper limit $F(>140 \mathrm{GeV})<1.6 \times 10^{-11} \mathrm{~cm}^{-2} \mathrm{~s}^{-1}$. The source has been monitored in the $R$-band by the Tuorla blazar monitoring program ever since.

B3 2247+381 was also included in the list of potentially interesting $\mathrm{TeV}$ sources released to the Imaging Atmospheric Cherenkov Telescope experiments by the Fermi-LAT collaboration on 27th October 2009 (Fermi-LAT Collaboration 2009, priv. comm.). B3 $2247+381$ is listed in the first Fermi-LAT catalog of AGNs (Abdo et al. 2010) as 1FGL J2250.1+3825 with a very hard spectrum (spectral index of $-1.6 \pm 0.1$ ). In the second Fermi-LAT catalog this object is listed as 2FGL $\mathrm{J} 2250+3825$ with a spectral index of $-1.84 \pm 0.11$ (Ackerman et al. 2012). Neronov et al. (2010) found a hint of signal at $2.73 \sigma$ in the Fermi-LAT data above $100 \mathrm{GeV}$ over the period August 2008-April 2010.

In this paper we present the first detection of VHE $\gamma$-ray emission triggered by the optical high state of the source and the first optical light curve of B3 2247+381. We also present simultaneous X-ray data obtained by the Swift and high energy (HE) data obtained by Fermi-LAT satellites.

\section{Observations and data analysis}

\subsection{MAGIC observations and data analysis}

The VHE $\gamma$-ray observations were carried out with the MAGIC telescopes located on the Canary Island of La Palma $\left(28.8^{\circ} \mathrm{N}\right.$, $17.8^{\circ} \mathrm{W}$ at $2200 \mathrm{~m}$.a.s.1). The two $17 \mathrm{~m}$ telescopes use the atmospheric Cherenkov imaging technique and allow for measurements at a threshold as low as $50 \mathrm{GeV}$.

B3 2247+381 was observed with the MAGIC telescopes during 13 nights between September 30th and October 30th 2010 collecting a total of $21.2 \mathrm{~h}$ of data, of which $5.3 \mathrm{~h}$ were discarded based on the event rate. The effective time of this observation, correcting for the dead time of the trigger and readout systems is $14.2 \mathrm{~h}$. Part of the data was taken under moderate moonlight conditions.

All the data were taken in the false-source tracking (wobble) mode (Fomin et al. 1994), in which the telescope was alternated every 20 min between two sky positions at $0.4^{\circ}$ offset from the source. The zenith angle was between $8^{\circ}$ and $35^{\circ}$.

The data was analysed using the standard MAGIC analysis framework "MARS" as described in Moralejo et al. (2009) with additional adaptations incorporating the stereoscopic observations. The images were cleaned using timing information as described in Aliu et al. (2009) with absolute cleaning levels of 6 phe (so-called "core pixels") and 3 phe ("boundary pixels") for the first telescope and 9 phe and 4.5 phe respectively for the second telescope. The images were parameterized in each telescope separately following the prescription of Hillas (1985).

\footnotetext{
${ }^{2}$ Note, that Veron-Cetty \& Veron (2006) list the source as a BL Lac candidate and that the classification has not been confirmed.

3 The stacked dataset of all X-ray selected blazars resulted in a significant $\gamma$-ray excess, which hints towards the fact that the sources were emitting at a very low level.
}

In order to reconstruct the shower arrival direction we used the random forest regression method (RF DISP method, Aleksic et al. 2010) which was extended using stereoscopic information such as the height of the shower maximum and the impact distance of the shower on the ground. We estimated the RF DISP for each telescope separately and obtained two possible solutions along the major axis of the shower image in each telescope, respectively. Finally, we searched for the combination of two solutions (one from each telescope) that have the shortest squared angular distance between them. If this squared angular distance is greater than 0.05 degree $^{2}$ the event is removed from further analysis. This improves the background rejection since hadron induced showers have a larger error on the reconstruction of the arrival direction. The final arrival direction is the mean of the two solutions (weighted by the number of pixels in each shower image).

For the gamma-hadron separation the random forest method is used (Albert et al. 2008b). In the stereoscopic analysis image parameters of both telescopes are used as well as the shower impact point and the shower height maximum. A detailed description of the stereoscopic MAGIC analysis can be found in (Aleksic et al., in prep.).

\subsection{Optical observations and data analysis}

B3 2247+381 has been observed by the Tuorla group ${ }^{4}$, using the $35 \mathrm{~cm}$ KVA telescope, located on La Palma, since 2006 (see Aleksić et al. 2011a for a description of the telescope). Observations have been made in the $R$-band. The brightness of the object was measured based on stars in the same CCDframes as B3 $2247+381$. These stars were calibrated by the Tuorla group during the observing seasons. $R$-band magnitudes were converted to fluxes, using: $F(\mathrm{Jy})=3080 \times 10^{-m_{R} / 2.5}$. The fluxes were corrected for galactic absorption by $R=$ 0.398 mag (Schlegel et al. 1998). During the years 2006-2009 B3 2247+381 was a quite faint and steady source at $R \sim 1.8 \mathrm{mJy}$, but during late summer 2010 it went to a high optical state, reaching an average flux level of $2.4 \mathrm{mJy}$. The source also stayed at this level throughout the observing season (see Fig. 3). In late September an alert was given to MAGIC and it started observations on September 30th. An alert is issued when the optical flux has increased by $50 \%$ from the long term running average.

\subsection{Swift observations and data analysis}

The prime objective of the Swift Gamma-Ray Burst observatory, launched in November 2004 (Gehrels et al. 2004), was to detect and follow up on Gamma-Ray bursts, but it has turned into a multi-purpose observatory due to its fast slewing and response capacity and its multi-wavelength coverage. Swift is equipped with three telescopes, the Burst Alert Telescope (BAT; Barthelmy 2005), which covers the $15-150 \mathrm{keV}$ range, the X-ray telescope (XRT; Burrows et al. 2005) covering the $0.3-10 \mathrm{keV}$ energy band, and the UV/Optical Telescope (UVOT; Roming et al. 2005) covering the 1800-6000 $\AA$ wavelength range.

Swift Target of Opportunity observations were requested and from October, 5 to 16, 2010. Swift/XRT observed the source for $\sim 5 \mathrm{ks}$ every night, in Photon counting (PC) mode. We also analysed Swift archival data from August 10th, 2009, February 18th, 2010 and April 18th, 2010 in order to compare the level of the X-ray emission to previous observations.

\footnotetext{
${ }^{4}$ http://users.utu.fi/kani/
} 
The data were processed with standard procedures using the FTOOLS task XRTPIPELINE (version 0.12.6) distributed by HEASARC within the HEASoft package (v.6.10). Events with grades 0-12 were selected for the PC data (see Burrows et al. 2005) and the response matrices available in the Swift CALDB (v.20100802) were used. For the spectral analysis, we extracted the PC source events in the $0.3-10 \mathrm{keV}$ range within a circle with a radius of 20 pixels $(\sim 47$ arcsec). The background was extracted from an off-source circular region of 40-pixels radius.

The spectra were extracted from the corresponding event files and binned using GRPPHA to ensure a minimum of 30 counts per bin in a manner so that the $\chi^{2}$ statistic could reliably be used. Spectral analyses were performed using XSPEC version 12.6.0. We adopted both a simple power law model and a log-parabolic model as in Massaro et al. (2004) with an absorption hydrogen-equivalent column density fixed to the Galactic value in the direction of the source, namely $1.2 \times 10^{21} \mathrm{~cm}^{-2}$. The two models provide similar results in terms of goodness of fit above $\sim 0.7 \mathrm{keV}$. However below this energy the differences are in general negligible due to low statistics.

Swift/UVOT observed the source in the "filter of the day" mode, that is a different filter was used for different observations. This does not allow to compare the UV fluxes among different days. UVOT source counts were extracted from a circular region 5 arcsec-sized centered on the source position, while the background was extracted from a larger circular nearby source free region. These data were processed with the uvotmaghist task of the HEASOFT package. The observed magnitudes have been corrected for Galactic extinction $E_{B-V}=0.149 \mathrm{mag}$ (Schlegel et al. 1998).

\subsection{Fermi data analysis}

The Fermi-LAT is a pair conversion telescope designed to cover the energy band from $20 \mathrm{MeV}$ to greater than $300 \mathrm{GeV}$ (Atwood et al. 2009) which operates in survey mode, scanning the entire sky every three hours. The data used in this paper encompasses the time interval from August 5th, 2008 to April 7th, 2011 (MJD 54683-55658), and were analyzed with the Fermi Science Tools package version v9r23p0, which are available from the Fermi Science Support Center (FSSC). Only events with the highest probability of being photons, those in the diffuse class, located within $12^{\circ}$ of B3 $2247+304$ were used in this analysis. In addition, a cut on the maximum zenith angle $\left(<100^{\circ}\right)$ was applied to reduce the contamination from the Earth-limb gamma-rays, which is produced by cosmic-rays interacting with the upper atmosphere. The background model used to extract the $\gamma$-ray signal includes a Galactic diffuse emission component and an isotropic component (including residual instrument background), modelled with the files gll_iem_v02_P6_V11_DIFFUSE.fit and isotropic iem_v02_P6_V11_DIFFUSE.txt, which are publicly available ${ }^{5}$. The normalizations of the components comprising the total background model were allowed to vary freely during the spectral point fitting. The spectral fluxes were derived with the postlaunch instrument response functions (IRF) P6_V11_DIFFUSE, and applying an unbinned maximum likelihood technique (Mattox et al. 1996) to events in the energy range spanning from $300 \mathrm{MeV}$ to $300 \mathrm{GeV}$. All the sources from the 2FGL catalog (Abdo et al. 2011) located within $7^{\circ}$ radius of B3 2247+38 were included in the model of the region. The initial parameters in

\footnotetext{
5 http://fermi.gsfc.nasa.gov/ssc/data/access/lat/ BackgroundModels.html
}

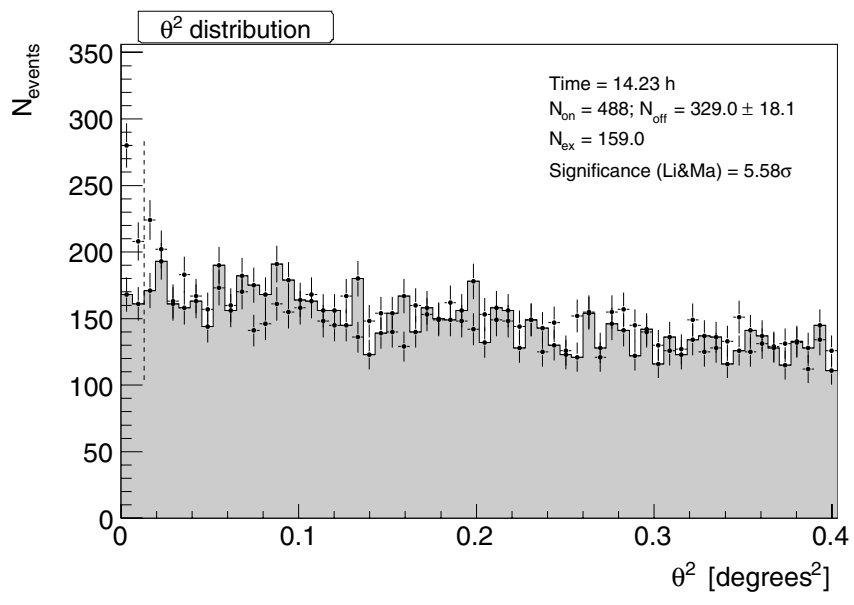

Fig. 1. Distribution of the squared angular distance $\left(\theta^{2}\right)$ for the onsource counts in the direction of B3 2247+381 (black points with errorbars) and the normalized off-source events (gray histogram).

the XML file were set to those of the 2FGL catalog, leaving the normalization parameters free in the fitting procedure. The systematic uncertainty in the flux is estimated as $10 \%$ at $100 \mathrm{MeV}$, $5 \%$ at $560 \mathrm{MeV}$ and $20 \%$ at $10 \mathrm{GeV}$ and above ${ }^{6}$.

For the period of the MAGIC observations (30 days between September 30th and October 30th 2010), the source is not significantly resolved, and hence only $95 \%$ confidence level upper limits were obtained. In the light curve presented in Fig. 3, for each time bin, if the $T S$ value for the source was $T S<4$ or the number of predicted photons $N_{-}$pred $<3$, the values of the fluxes were replaced by the $95 \%$ confidence level upper limits. The 2-sigma upper limits were computed using the Bayesian method (Helene 1983), where the likelihood is integrated from 0 up to the flux that encompasses $95 \%$ of the posterior probability.

\section{Results}

In the MAGIC data the distribution of the square of the angle $\theta$ between the reconstructed direction of the events after cuts and the position of B3 2247+381 (RA: 22.83472 ${ }^{\circ}$, Dec: $38.41028^{\circ}$, J2000 as in Ficarra et al. 1985) shows an excess of $159 \pm 28$ events above a threshold of $200 \mathrm{GeV}$. The cuts were previously optimized on a trial sample of Crab Nebula data. The threshold was calculated from Monte Carlo simulated data by finding the peak of the differential rate vs. energy distribution after cuts and spectral re-weighting. The expected background level with the same cuts is 329 events, calculated from the $\theta^{2}$ distribution of the reconstructed direction of the events with respect to the anti-source position, located $180^{\circ}$ from the real source position in the camera plane (Fig. 1). The measured excess corresponds to a post-trial significance of $5.6 \sigma$ calculated using Eq. (17) from Li \& Ma (1983).

The source position and extension, determined by a 2D Gaussian fit of the sky map produced with the cuts above, are consistent with a point-like source placed at the position of B3 $2247+381$ within $0.015^{\circ}$, well within the statistical uncertainty and the systematic pointing uncertainties of MAGIC.

The integral flux of the source above $200 \mathrm{GeV}$ was estimated to be $\left(5.0 \pm 0.6_{\text {stat }} \pm 1.1_{\text {sys }}\right) \times 10^{-12} \mathrm{ph} \mathrm{cm}^{-2} \mathrm{~s}^{-1}$. The differential energy spectrum is well described by a simple powerlaw: $\mathrm{d} N / \mathrm{d} E=f_{0}(E / 300 \mathrm{GeV})^{\gamma}$. The photon index $\gamma$ was found

\footnotetext{
6 http://fermi.gsfc.nasa.gov/ssc/data/analysis/ LAT_caveats.html
} 


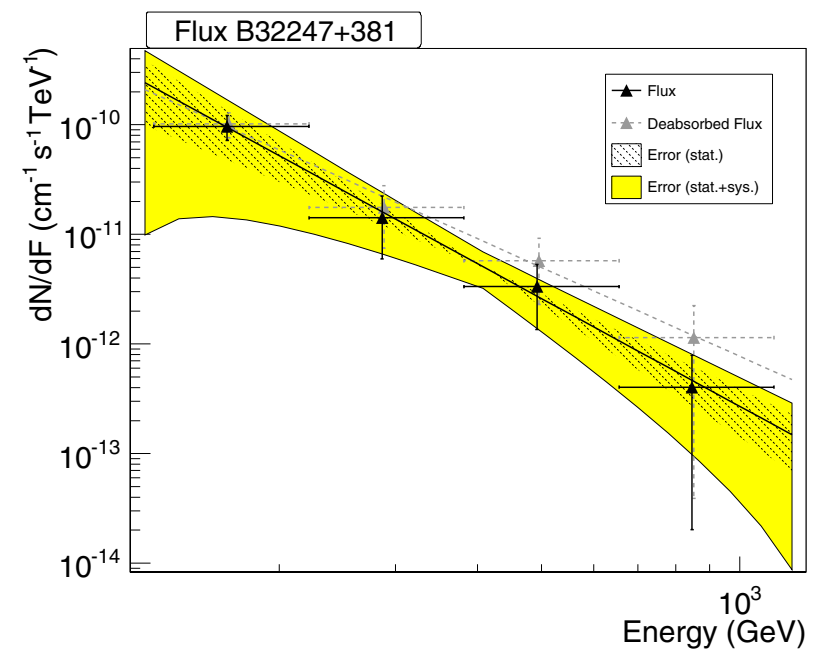

Fig. 2. The unfolded differential energy spectrum of B3 2247+381 observed by MAGIC. The black data points refer to the measured spectrum, while the grey dashed points have been corrected for the attenuation of the EBL. The thick black and dashed grey lines are power-law fits to the respective data points (fit results are given in the text). The dashed band corresponds to the statistical error of the fit to the measured spectrum, while the white band surrounding it is the sum of the statistical and systematic errors of the fit.

to be $-3.2 \pm 0.5_{\text {stat }} \pm 0.5_{\text {sys }}$, and a flux normalization $f_{0}$ at $300 \mathrm{GeV}$ of $\left(1.4 \pm 0.3_{\text {stat }} \pm 0.2_{\text {sys }}\right) \times 10^{-11} \mathrm{ph} \mathrm{cm}^{-2} \mathrm{~s}^{-1} \mathrm{TeV}^{-1}$. In order to correct the effects in the spectrum determination introduced by the limited energy resolution, different unfolding algorithms (Forward, Tikhonov, Schmelling 1\&2, Bertero; all described in Albert et al. 2007b) were used, and all agreed within errors. Taking into account the attenuation due to pair production with the extragalactic background light (EBL), the spectrum was found to be compatible with a power law with photon index $\gamma=-2.7 \pm 0.5_{\text {stat }} \pm 0.5_{\text {sys }}$ and flux at $300 \mathrm{GeV}$ $f_{0}=\left(2.0 \pm 0.3_{\text {stat }} \pm 0.3_{\text {sys }}\right) \times 10^{-11} \mathrm{ph} \mathrm{cm}^{-2} \mathrm{~s}^{-1} \mathrm{TeV}^{-1}$ (Fig. 2). Two different EBL models were used (Domínguez et al. 2011; and Kneiske \& Dole 2010), and they were found to be in good agreement with each other, well within the statistical uncertainties.

Long term light curves of B3 $2247+381$ in VHE $\gamma$-rays (MAGIC), HE $\gamma$-rays (Fermi-LAT), X-rays (Swift) and optical (Tuorla Observatory) are shown in Fig. 3. Our detection in VHE $\gamma$-rays is compatible with the previous upper limit from 2006 and thus no variability can be established in this energy band. However, in X-rays and the optical band a clear increase of the flux in fall 2010 is evident, while the Fermi-LAT light curve is consistent with a constant flux. A fit with a constant to the eleven Fermi flux points where the source is significantly detected, gave a flux value of $(3.7 \pm 0.5) \times 10^{-9} \mathrm{ph} \mathrm{cm}^{-2} \mathrm{~s}^{-1}$, with a reduced $\chi^{2}$ of 0.7 with ten degrees of freedom. The Fermi-LAT is not sensitive enough for detecting short term variations at this flux level. The temporal evolution of the VHE $\gamma$-ray, X-ray and optical emission from B3 2247+381 during September-October 2010 observation shows no strong variability on time scales of a night (Fig. 4). In particular, the MAGIC light curve above $200 \mathrm{GeV}$ is consistent with a non-variable source, having a reduced $\chi^{2}$ of 0.6 with ten degrees of freedom. During one night the X-ray flux is significantly higher (almost factor 2) than the other X-ray points, but unfortunately we do not have simultaneous optical or MAGIC data for that night.
Time[date]
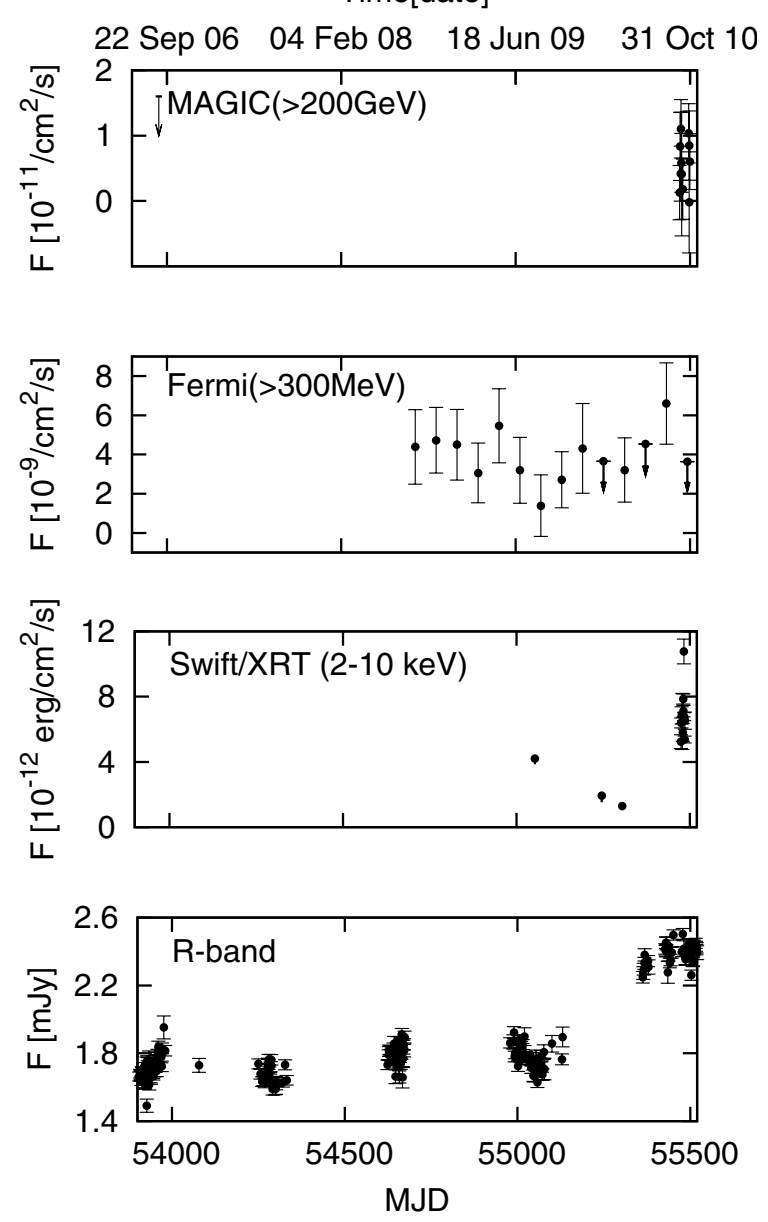

Fig. 3. Long term light curves of B3 $2247+381$ in VHE $\gamma$-rays, FermiLAT HE $\gamma$-rays (two months time intervals), Swift X-rays and optical KVA $R$-band.

\section{Discussion}

In this paper we report the discovery of VHE $\gamma$-rays from B3 $2247+381$ by MAGIC. The MAGIC observations were triggered by an optical high state of the source, like several other discoveries. However, the observed VHE $\gamma$-ray flux is consistent with the upper limit from previous observations and we therefore cannot conclude if the source was in a higher VHE $\gamma$-ray state during the observations.

In Fig. 5 we show the spectral energy distribution of the source during the MAGIC observations, together with simultaneous Swift and optical data, and other non-simultaneous data. The Swift observations show that the source was in a high state also in X-rays. In the Fermi energy range the source is very weak, which limits the capability of detecting statistically significant flux-variability on time scales of a few months. The synchrotron component of the SED is showing a significantly larger emission in the high state, while the inverse Compton component is consistent with only minor changes. We must however note that the weak detection in the HE and VHE $\gamma$-ray band significantly limits the determination of the inverse Compton component.

We reproduce the SED with a one-zone synchrotron-selfCompton (SSC) model (see Tavecchio et al. 2001, for a description). In brief, the emission region is assumed to be spherical, with a radius $R$, filled with a tangled magnetic field of intensity $B$. The relativistic electrons follow a smoothed broken 
Table 1. Input parameters for the high and low states of the SSC-model shown as solid and dashed lines in Fig. 5.

\begin{tabular}{lccccccccc}
\hline \hline Flux state & $\gamma_{\min }$ & $\gamma_{\mathrm{b}}$ & $\gamma_{\max }$ & $n_{1}$ & $n_{2}$ & $\begin{array}{c}B \\
\mathrm{G}\end{array}$ & $\begin{array}{c}K \\
\mathrm{~cm}^{-3}\end{array}$ & $\begin{array}{c}R \\
\mathrm{~cm}\end{array}$ \\
\hline High & $3 \times 10^{3}$ & $7.1 \times 10^{4}$ & $6 \times 10^{5}$ & 2.0 & 4.35 & 0.06 & $2.5 \times 10^{3}$ & 35 & $8 \times 10^{15}$ \\
Low & $3 \times 10^{3}$ & $6.8 \times 10^{4}$ & $5 \times 10^{5}$ & 2.0 & 5.35 & 0.08 & $1.15 \times 10^{4}$ & 30 & $4 \times 10^{15}$ \\
\hline
\end{tabular}

Notes. For more explanations see text.
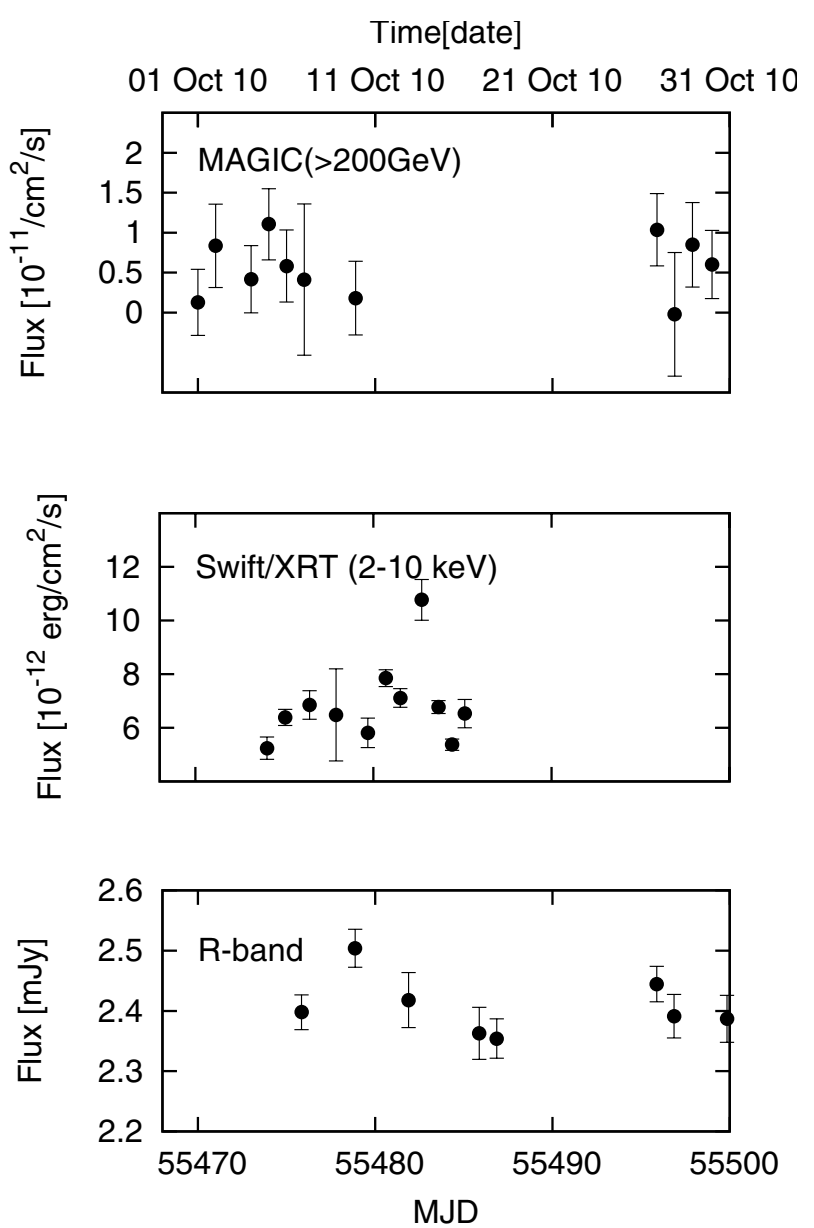

Fig. 4. Same as Fig. 3, but zoomed into the time interval of the MAGIC observations in September-October 2010.

power-law energy distribution specified by the limits $\gamma_{\min }, \gamma_{\max }$ and the break at $\gamma_{\mathrm{b}}$ as well as the slopes $n_{1}$ and $n_{2}$ before and after the break, respectively. Relativistic effects are taken into account by the Doppler factor $\delta$. The used input model parameters are shown in Table 1.

To reproduce the change of the Compton and synchrotron luminosity ratio between the low and the high state we mainly act on the electron normalization, source radius and Doppler factor (with slight changes also to the other parameters). The steeper $\mathrm{X}$-ray slope in the low state implies a larger value of $n_{2}$.

The comparison with parameters derived for BL Lac objects (e.g. Tavecchio et al. 2010) reveals that the parameters used for B3 $2247+381$ are close to the typical values. As for other sources, the somewhat larger (lower) value of the Doppler factor $\delta$ (the magnetic field intensity $B$ ) with respect to "standard" values is mainly due to the relatively large separation between the synchrotron and IC peaks.

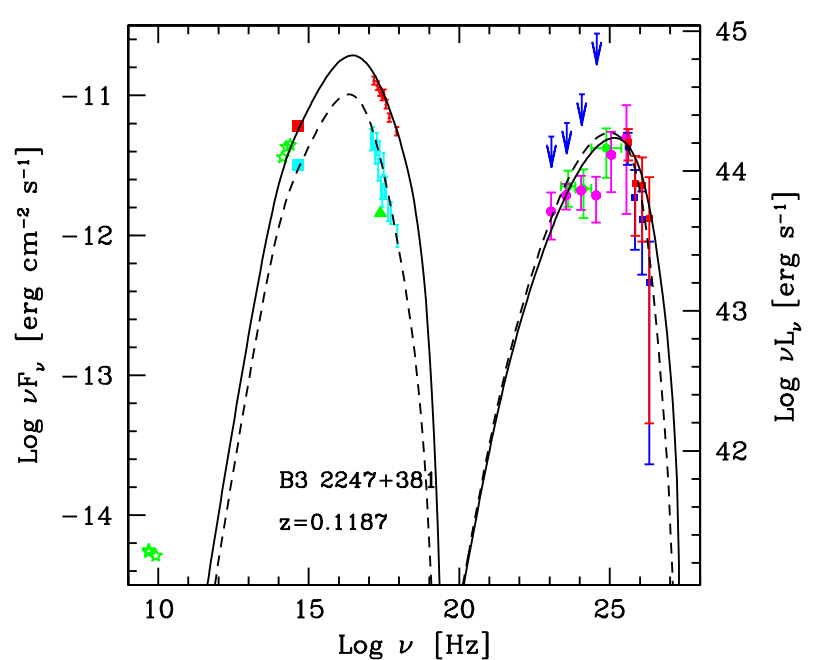

Fig. 5. Spectral energy distribution of B3 2247+381 (red: EBL corrected MAGIC spectral points). The green crosses are 1FGL Fermi data points (Abdo et al. 2010), while the pink points represent the Fermi analysis from this work (2.5 years of data). Blue arrows show the $95 \%$ confidence upper limits computed from Fermi-LAT data for the time interval of the MAGIC observation. Low (high) state Swift data were taken on April 18th 2010 (October 5-16, 2010). The host galaxy contribution has been subtracted from the KVA $R$-band data (red and light blue squares), following Nilsson et al. (2007). The data have been corrected for galactic absorption. Green and light blue points represent non-simultaneous low state data. The solid line is our SSC-model fit to the high state observations; the dotted line is a fit to the low state observations.

Additionally, we model the SED, during the high state, with the one-zone SSC code from Weidinger et al. (2010) which is shown in Fig. 6. In contrast to the model from Tavecchio et al. (2001) all parameters are basic physical parameters and the electron and photon spectrum and their breaks are derived self-consistently with a continuous injection of monochromatic electrons at the energy $\gamma_{0}=10^{4}$ and injection rate $K=8.4 \times$ $10^{4} \mathrm{~cm}^{-3} \mathrm{~s}^{-1}$. The spectrum is the resulting steady-state solution. The environment is defined by the magnetic field $B=0.07 \mathrm{G}$, the acceleration efficiency $t_{\mathrm{acc}} / t_{\mathrm{esc}}=1.09$ (i.e. the particle spectral index is $s=2.09$ and the resulting $\gamma_{\max }=4.8 \times 10^{5}$ ), and the blob radius $R=1.3 \times 10^{16} \mathrm{~cm}$. The break in the $\mathrm{e}^{-}$spectrum of 1 at $\gamma_{b}=2.9 \times 10^{4}$ arises self-consistently from IC and synchrotron cooling. The common parameters of both models agree very well.

The optical monitoring of candidate VHE $\gamma$-ray blazars has proven to be a successful tool for their discovery. However, for B3 2247+381 (like for Mrk 180 and 1ES 1011+496) we cannot establish a connection between the optical high state and the VHE $\gamma$-ray emission since the upper limit from previous observations (during a low optical state) is higher than the detected VHE $\gamma$-ray flux during the discovery. Therefore further 
A\&A 539, A118 (2012)

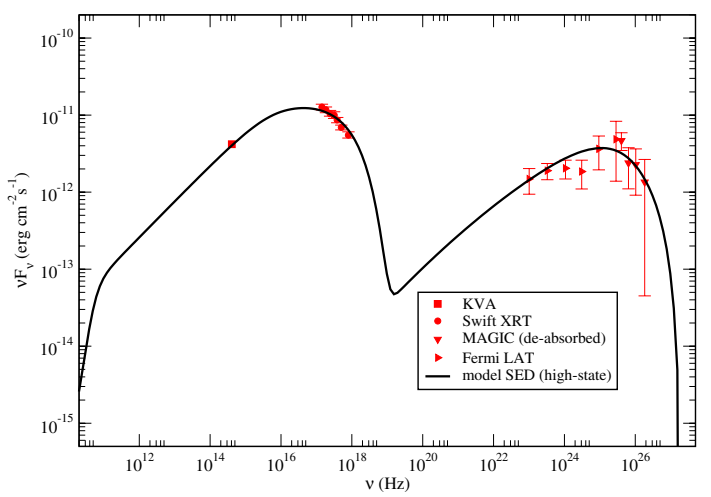

Fig. 6. The solid line shows the SED model using Weidinger et al. (2010). The data points are described in the inlay of the figure. The fit parameters can be found in the text.

observations are still needed to study the connection between these two wavebands (see also Reinthal et al. 2011).

Acknowledgements. We would like to thank the Instituto de Astrofísica de Canarias for the excellent working conditions at the Observatorio del Roque de los Muchachos in La Palma. The support of the German BMBF and MPG, the Italian INFN, the Swiss National Fund SNF, and the Spanish MICINN is gratefully acknowledged. This work was also supported by the Marie Curie program, by the CPAN CSD2007-00042 and MultiDark CSD2009-00064 projects of the Spanish Consolider-Ingenio 2010 programme, by grant DO02-353 of the Bulgarian NSF, by grant 127740 of the Academy of Finland, by the YIP of the Helmholtz Gemeinschaft, by the DFG Cluster of Excellence "Origin and Structure of the Universe", and by the Polish MNiSzW Grant N N203 390834. The Fermi-LAT Collaboration acknowledges support from a number of agencies and institutes for both development and the operation of the LAT as well as scientific data analysis. These include NASA and DOE in the United States, CEA/Irfu and IN2P3/CNRS in France, ASI and INFN in Italy, MEXT, KEK, and JAXA in Japan, and the K. A. Wallenberg Foundation, the Swedish Research Council and the National Space Board in Sweden. Additional support from INAF in Italy and CNES in France for science analysis during the operations phase is also gratefully acknowledged. We gratefully acknowledge N. Gehrels for approving this set of ToOs and the entire Swift team, the duty scientists and science planners for the dedicated support, making these observations possible.

\section{References}

Abdo, A. A., Ackermann, M., Ajello, M., et al. 2010, ApJ, 715, 429 Abdo, A. A., et al. (Fermi-LAT Collaboration) 2011, ApJS, submitted [arxiv: 1108.1435]

Aharonian, F., Akhperjanian, A. G., Anton, G., et al. 2009, ApJ, 696, L150

Ackerman, M., et al. 2012, ApJ, in press

Albert, J., Aliu, E., Anderhub, H., et al. 2006, ApJ, 648, L105

Albert, J., Aliu, E., Anderhub, H., et al. 2007a, ApJ, 667, L21

Albert, J., Aliu, E., Anderhub, H., et al. 2007b, Nucl. Instr. Meth. A, 583, 494

Albert, J., Aliu, E., Anderhub, H., et al. 2008a, Science, 320, 1752

Albert, J., Aliu, E., Anderhub, H., et al. 2008b, Nucl. Instr. Meth. A, 588, 424

Aleksić, J., Anderhub, H., Antonelli, L. A., et al. 2010, ApJ, 519, A32

Aleksić, J., Antonelli, L. A., Antoranz, P., et al. 2011a, A\&A, 530, A4

Aleksić, J., Antonelli, L. A., Antoranz, P., et al. 2011b, ApJ, 729, 115

Aliu, E., Anderhub, H., Antonelli, L. A., et al. 2009, ApJ, 692, L29

Anderhub, H., Antonelli, L. A., Antoranz, P., et al. 2009, ApJ, 704, L129

Atwood, W. B., Abdo, A. A., Ackermann, M., et al. 2009, ApJ, 697, 1071

Barthelmy, S. D. 2005, Space Sci. Rev., 120, 143

Burrows, D. N., Hill, J. E., Nousek, J. A., et al. 2005, Space Sci. Rev., 120, 165 Domínguez, A., Primack, J. R., Rosario, D. J., et al. 2011, MNRAS, 410, 2556 Donato, D., Ghisellini, G., Tagliaferri, G., \& Fossati, G. 2001, A\&A, 375, 739

Falco, E. E., Kochanek, C. S., \& Munoz, J. A. 1988, ApJ, 494, 47

Ficarra, A., Grueff, G., \& Tomassetti, G. 1985, A\&AS, 59, 255

Fomin, V. P., Stepanian, A. A., Lamb, R. C., et al. 1994, ApJ, 2, 137

Foschini, L., Ghisellini, G., Tavecchio, F., et al. 2007, ApJ, 657, 81

Gehrels, N., Chincarini, G., Giommi, P., et al. 2004, ApJ, 611, 1005
Helene, O. 1983, Nucl. Instr. Meth. Phys. Res., 212, 319

Hillas, A. M. 1985, Proc. of the 19th ICRC, 3, 445

Kneiske, T. M., \& Dole, H. 2010, A\&A, 515, A19

Li, T.-P., \& Ma, Y.-Q. 1983, ApJ, 272, 317

Mariotti, M. 2011, Atel \# 3100

Mariotti, M., MAGIC Collaboration 2010, Atel \# 2910

Massaro, E., Perri, M., Giommi, P., \& Nesci, R. 2004, A\&A, 413, 489

Mattox, J. R., Bertsch, D. L., Chiang, J., et al. 1996, ApJ, 461, 396

Moralejo, A., Gaug, M., Carmona, E., et al. 2009, in Proc. 31st ICRC (Lodz) [arXiv: 0907.0943]

Neronov, A., Semikoz, D. V., \& Vovk, I. 2011, A\&A, 529, A59

Nieppola, E., Tornikoski, M., \& Valtaoja, E. 2006, A\&A, 445, 441

Nilsson, K., Pasanen, M., Takalo, L. O., et al. 2007, A\&A, 475, 199

Reinthal, R., et al. 2011, Proc. Beamed and Unbeamed Gamma-rays from Galaxies, Open Access Journal of Physics: Conference Series (JCPS), in press

Roming, P. W. A., Kennedy, T. E., Mason, K. O., et al. 2005, Space Sci. Rev., 120,95

Schlegel, D. J., Finkbeiner, D. P., \& Davis, M. 1998, ApJ, 500, 525

Tavecchio, F., Maraschi, L., Pian, E., et al. 2001, ApJ, 554, 725

Tavecchio, F., Ghisellini, G., Ghirlanda, G., Foschini, L., \& Maraschi, L. 2010, MNRAS, 401, 1570

Veron-Cetty, M. P., \& Veron, P. 2006, A\&A, 455, 773

Weidinger, M., Rüger, M., \& Spanier, F. 2010, ASTRA, 6, 1

1 IFAE, Edifici Cn., Campus UAB, 08193 Bellaterra, Spain e-mail: gtto@ifae.es

2 Universidad Complutense, 28040 Madrid, Spain

3 INAF National Institute for Astrophysics, 00136 Rome, Italy

4 Università di Siena, and INFN Pisa, 53100 Siena, Italy

5 Technische Universität Dortmund, 44221 Dortmund, Germany

6 Università di Padova and INFN, 35131 Padova, Italy

7 Inst. de Astrofísica de Canarias, 38200 La Laguna, Tenerife, Spain e-mail: berger@astro.uni-wuerzburg.de

8 Depto. de Astrofísica, Universidad de La Laguna, 38206 La Laguna, Spain

9 University of Łódź, 90236 Lodz, Poland

10 Tuorla Observatory, University of Turku, 21500 Piikkiö, Finland e-mail: takalo@utu.fi

11 Deutsches Elektronen-Synchrotron (DESY), 15738 Zeuthen, Germany

12 ETH Zurich, 8093 Zurich, Switzerland

13 Max-Planck-Institut für Physik, 80805 München, Germany

14 Universität Würzburg, 97074 Würzburg, Germany

15 Universitat de Barcelona (ICC/IEEC), 08028 Barcelona, Spain

16 Università di Udine, and INFN Trieste, 33100 Udine, Italy

17 Institut de Ciències de 1'Espai (IEEC-CSIC), 08193 Bellaterra, Spain

18 Inst. de Astrofísica de Andalucía (CSIC), 18080 Granada, Spain

19 Croatian MAGIC Consortium, Rudjer Boskovic Institute, University of Rijeka and University of Split, 10000 Zagreb, Croatia

20 Universitat Autònoma de Barcelona, 08193 Bellaterra, Spain

21 Inst. for Nucl. Research and Nucl. Energy, 1784 Sofia, Bulgaria

22 INAF/Osservatorio Astronomico and INFN, 34143 Trieste, Italy

23 Università dell'Insubria, Como, 22100 Como, Italy

24 Università di Pisa, and INFN Pisa, 56126 Pisa, Italy

25 ICREA, 08010 Barcelona, Spain

26 Now at: École polytechnique fédérale de Lausanne (EPFL), Lausanne, Switzerland

27 supported by INFN Padova

28 Now at: Centro de Investigaciones Energéticas, Medioambientales y Tecnológicas (CIEMAT), Madrid, Spain

29 Now at: Finnish Centre for Astronomy with ESO (FINCA), University of Turku, Finland

30 Istituto Nazionale di Fisica Nucleare, Sezione di Padova, 35131 Padova, Italy

31 Dipartimento di Fisica G. Galilei, Università di Padova, 35131 Padova, Italy 